\title{
The Long Correlation Option Pricing based on Binary Tree Model
}

\author{
Gui-Bin $\mathrm{HU}^{1, \mathrm{a}}$, Rui-Jia ZHANG ${ }^{1, b}$, Xiang-Xing TAO ${ }^{2, \mathrm{c}}$ \\ ${ }^{1}$ Ningbo university, Ningbo, Zhejiang, 315211, China \\ ${ }^{2}$ Zhejiang Institute of Science and Technology, Hangzhou, Zhejiang, 310023 China \\ 15557116489@126.com
}

\begin{abstract}
Keywords: Binary Tree, No Arbitrage, Markov Column, Long Correlation, European Call Option.
\end{abstract}
\begin{abstract}
In order to price stock option better, it gets a new process of stock price which has a long correlation of binary tree based on classic financial random binary tree with no-arbitrage asset pricing model. We think investors drive the price of the stock and suppose that the premise of stock price which don't obey Markov column. And we price option of stuck by Matlab 7.0( a computer software). So it also gets a new pricing method on European stock call option. In this way, we can price other option better.
\end{abstract}

\section{Introduction}

Stock is a kind of securities, which is a special financial security given by joint-stock company. Meanwhile, the company can raise funds or holders can get stock dividend and bonus from it. The stock is a basic and important method for the corporation to raise funds or investor to invest. Under this circumstance, all kinds of derivative come out following stock market, such as, stocks, futures, Stock options, Among them, stock option boosts the financial market development greatly. As a result, it is long time to do research on the significance and applicability of option pricing.

The contract of option pricing is an optional product. The holder have the rights but not obligations to buy or sell the stock at the appoint price in the certain days of future.According the risk transfer principle, the holder of stock option have the advantage to sell it at the profit environment, meanwhile, the risk transfer to the seller. Hence, the buyer need pay amount of budget to the option seller. The two sides can reach a valid transaction.

So the pricing of option is a key progress. The changing of stock price have directly influence on option pricing. In order to price the option, we can make a binary tree modal in common. The theory mainly hypothesis that the stock price submit to random walk theory and Brownian motion and accord with non-arbitrage hypothesis, and the process of changing follow Markov column. It means that the stock price will present binary tree graph among option pricing period. The future stock price will increase at certain proportionality based on stock price now, and decrease at certain proportionality along with another random probability. However, according to the stock price of the real inner drive, the price of the stock is not a Markov random process. At the same time a large number of empirical results also confirmed that the stock price process is not subject to the standard Brownian motion. So this article gives a random process of stock prices which have long correlation of binary tree on the basis of the classical binary tree model and it also gets a European call option pricing method.

\section{The Proposed Correlation Length Binary Stock Price Model}

The binary asset pricing model had already proposed by Cox, Ross and Robinstein [1] and Fischer Black and Myron Scholes [2] in 1979, and it was studied more deeper in some financial books [3-4]. Meanwhile, some scholars are also used for binary option pricing model [5]. Although traditional binomial model is simple, fast and effective, there are some problems. So there are some verifications and improvements of the binomial model by some scholars [6-7], and they had improved the binomial model from different angles. But they all missed the important point: binomial model is a simple the way where people look at the history of the stock for the long-term 
future of price correlation. It tells us that the random process of stock price is not a Markov chain, so it does not accurately reflect the movement of the stock price of stochastic processes. Because the reality of the stock price is driven directly by the investor, historical price records is an important factor which affect investors to judge the stock price, then the stock price would be brought by a variety effects pros and cons of historical price-related. So we have to consider the long-term impact on the very relevance of the historical price of the stock price. This paper is on the classical binary tree (Figure 1 is a two-step process of the classic binary random stock price.).The next presented below has proposed a long correlation stock price stochastic process models.

Firstly, there are some specific assumptions: the stock can be transaction by arbitrarily split; the rates of investment and borrowing are same, the market is frictionless, namely stock trading no difference; the random stock price has links with the stock price of historical on any time; all stock investors treat historical market prices on the future price of reading and understanding are different, but consistent is impaction.

Secondly, it constructs a new stock price model based on these assumptions. There is a stock which the risk-free interest rate is $r$ and the volatility rate of a non-dividend stocks is $\sigma$. Figure 1 is a classic two-step binomial model diagrams and figure 2 is a binary stock correlation length binary stock binomial model based on these two steps.

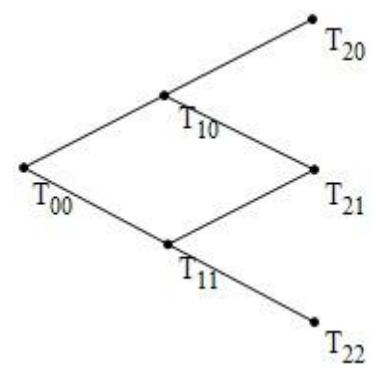

Fig1 Two-step diagram of a binary tree stock

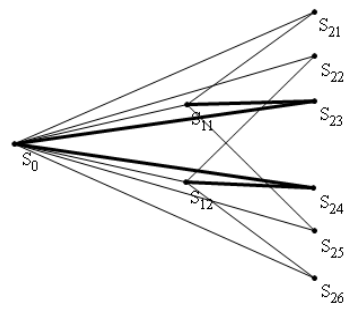

Fig 2 The two-step diagram of length correlation binary tree stock based on Fig 1

We can get a binary model of stock price mode which has long correlation of the random based on a two-step binary tree constructed from figure 2 .

If $T_{i j}$ represent the stock prices in classical binomial model, $i$ represents the number of step, $j$ represents the number of state. $S_{n, k}$ is a random variable, $b_{i}$ is a weights series. $i, j, k=0,1,2, \cdots$.

The random stock price based on Step 0 in the new model is

$$
S_{\mathrm{O} 1}=1 \times T_{\mathrm{OO}}
$$

The random stock prices based on Step 1 in the new model are:

$$
\left(\begin{array}{l}
S_{11} \\
S_{12}
\end{array}\right)=\left(\begin{array}{l}
b_{1} T_{10}+b_{0} T_{00}(1+r) \\
b_{1} T_{11}+b_{0} T_{00}(1+r)
\end{array}\right)
$$


The random stock prices based on Step 2 in the new model are:

$$
\left(\begin{array}{l}
S_{21} \\
S_{22} \\
S_{23} \\
S_{24} \\
S_{25} \\
S_{26}
\end{array}\right)=\left(\begin{array}{l}
b_{2} T_{20}+b_{1} T_{10}\left(1+\frac{r}{2}\right)+b_{0} T_{00}\left(1+\frac{r}{2}\right)^{2} \\
b_{2} T_{20}+b_{1} T_{11}\left(1+\frac{r}{2}\right)+b_{0} T_{00}\left(1+\frac{r}{2}\right)^{2} \\
b_{2} T_{21}+b_{1} T_{10}\left(1+\frac{r}{2}\right)+b_{0} T_{00}\left(1+\frac{r}{2}\right)^{2} \\
b_{2} T_{21}+b_{1} T_{11}\left(1+\frac{r}{2}\right)+b_{0} T_{00}\left(1+\frac{r}{2}\right)^{2} \\
b_{2} T_{22}+b_{1} T_{10}\left(1+\frac{r}{2}\right)+b_{0} T_{00}\left(1+\frac{r}{2}\right)^{2} \\
b_{2} T_{22}+b_{1} T_{11}\left(1+\frac{r}{2}\right)+b_{0} T_{00}\left(1+\frac{r}{2}\right)^{2}
\end{array}\right)
$$

When the number is increase, we can construct more binary tree stock price random variables, so it gets the following definition.

Definition: Suppose there is a $n$ steps binomial model from 0 to $T$, and $T_{i j}$ is a classic stock price, so $T_{n i}=S_{0} u^{i} d^{n-i}, u$ and $d$ are rising and falling proportion. then the new stock prices are met $S_{n, k}=\sum_{i=0,0 \leq a_{i} \leq i}^{n} b_{i} T_{i a_{i}}\left(1+\frac{r}{n}\right)^{(n-i) \Delta t}, k=\sum_{i=0}^{n} a_{i} \times(i !)+1,0 \leq a_{i} \leq i$, This model is called the long correlation binary model. $b_{i}$ represents a weight when its stock price in $t_{i}$ on the stock weighting of the stock price in classical binomial model, and $\sum_{i=0}^{n} b_{i}=1$.All investors look at the history of the stock price impact on the future relevance of the stock price. To facilitate the establishment of design models, we can suppose $b_{i}=\frac{1}{2^{n-i+1}}$.

If we use a matrix represent the stock price above stochastic process.

$$
\left(\begin{array}{l}
S_{n, 1} \\
\vdots \\
S_{n, k} \\
\vdots \\
S_{n,(n+1) !}
\end{array}\right)=S_{0}\left(\begin{array}{llll}
d^{n} & d^{n-1} & \cdots & 1 \\
\vdots & \vdots & \vdots & \vdots \\
u^{a_{n}} d^{n-a_{n}} & u^{a_{n-1}} d^{n-1-a_{n-1}} & \cdots & 1 \\
\vdots & \vdots & \vdots & \vdots \\
u^{n} & u^{n-1} & \cdots & 1
\end{array}\right)\left(\begin{array}{l}
\frac{1}{2}\left(1+\frac{r}{n}\right)^{0} \\
\vdots \\
\frac{1}{2^{i+1}}\left(1+\frac{r}{n}\right)^{i \Delta t} \\
\vdots \\
\frac{1}{2^{n+1}}\left(1+\frac{r}{n}\right)^{n \Delta t}
\end{array}\right)
$$

Also it is $S_{(n+1) \times 1}=S_{0} A_{(n+1) ! \times(i+1)} B$. Among it, $A$ is based on all possible combinations of random binomial model stock status values (because investor can not accurately know the relationship between them, so there are a variety of combinations. $B=\left(\frac{1}{2}\left(1+\frac{r}{n}\right)^{0} \frac{1}{4}\left(1+\frac{r}{n}\right)^{1 \Delta t} \quad \cdots \quad \frac{1}{2^{n+1}}\left(1+\frac{r}{n}\right)^{n \Delta t}\right)^{T}, \frac{1}{2^{i+1}}$ represents the market on the extent of the importance of the right to re-stock. From the definition above, we can get a random distribution of stock price. 
Tab. 1 Stock Price random probability distribution

\begin{tabular}{c|c}
\hline$S(n, k)$ & $\sum_{i=0}^{n} S_{0} u^{a_{i, j}} d^{i-a_{i, j}} b_{i}\left(1+\frac{r}{n}\right)^{(n-i) \frac{T}{n}}$ \\
\hline$P(n, k)$ & $\prod_{i=1}^{n}\left(\begin{array}{l}i \\
a_{i, k}\end{array}\right) p^{\sum_{i=1}^{n} a_{i, k}}(1-p)^{\frac{n(n+1)}{2}-\sum_{i=1}^{n} a_{i, k}}$ \\
\hline
\end{tabular}

(Note: Assuming every stock investor has different reviews on the importance of stock trends with historical stock price. For all possible stock binomial model, there are different combination.

Among them $0 \leq i \leq n, 0 \leq j \leq i, 0 \leq k \leq(n+1)$ ! and $0 \leq a_{i, k} \leq i . P(n, k)$ is the product of the probabilities of all of these combinations, $\left.\left(\begin{array}{l}n \\ m\end{array}\right)=\frac{n !}{m !(n-m) !}\right)$

\section{The Stock Option Pricing Model with Long Binary Correlation}

Lemma 1: There is a binary no-arbitrage model under the risk-neutral. We know that $p^{\prime} u+\left(1-p^{\prime}\right) d=1+r$, So we can get the risk-neutral probability rises $p^{\prime}=\frac{1+r-d}{u-d}$.

Lemma 2: There is a classical binary model which has $n$ steps and it meet the absence of arbitrage and martingale conditions we can know that: $E\left(S_{i} / T_{n}\right)=S_{0}\left(1+\frac{r}{n}\right)^{T}$.

Lemma 3: The stock price process is a martingale process under the same risk-neutral measure.

Suppose initial stock price of a non-dividend is $S_{0}$, the strike price is $K$, for a period is $T$, the market risk-free rate is $r$, volatility of the European call option is $\sigma$, and these amounts are constant. We can calculate the price of this European call option.

According to the definition, in order to get the option value of the options we must solve the following inequality

$$
S(n, k)=\sum_{i=0}^{n} S_{0} u^{a_{i, j}} d^{i-a_{i, j}} b_{i}\left(1+\frac{r}{n}\right)^{(n-i) \frac{T}{n}} \geq K
$$

We can get all of the solution by computer. So we can obtain the option value of a European call option price of this stock. So the price of a European call option $t$ is

$$
c=e^{-r T} E\left[\max \left(\left(S_{j}-K\right), 0\right)\right]
$$

We can carry out the price of European call option in this way.

In the same way, we can get the long European put stock option correlation theorem. Which is a long correlation binomial model based on the definition of European put stock option. We also can calculate European call or put option price by computer program.

\section{The Difference between Long Correlation Binary Tree Model and Other Pricing Formula}

Next we had compared some difference European bullis option pricing methods, and concluded 
the difference and interrelation between them. (1) Classical binary tree model method;

(2) Black-Scholes and Morton formula. (3) The simple Long correlation binary tree model. we assume $S_{0}=42$ dollar, $K=40$ dollar $r=0.1 \sigma=0.2, T=0.5$ year.

We get table 2 below by computer software programming

Tab. 2 Various stock option pricing methods for specific pricing

\begin{tabular}{ccccccccc}
\hline Pricing Method & Steps & $u$ & $d$ & $p$ & $q$ & $c$ & $E$ & $V$ \\
\hline \multirow{2}{*}{$\begin{array}{c}\text { Classical } \\
\text { binomial model }\end{array}$} & $n=30$ & 1.0262 & 0.9745 & 0.5258 & 0.4742 & 4.7496 & 44.155 & 0.1490 \\
& $n=40$ & 1.0226 & 0.9779 & 0.5224 & 0.4776 & 4.7538 & 44.155 & 0.1491 \\
& $n=50$ & 1.0202 & 0.9802 & 0.5200 & 0.4800 & 4.7640 & 44.153 & 0.1492 \\
\hline $\boldsymbol{B}$-S formula & $n=\infty$ & -- & -- & -- & -- & 4.7600 & 44.155 & 0.1414 \\
\hline & $n=6$ & 1.0594 & 0.9439 & 0.5580 & 0.4420 & 3.5001 & 43.416 & 0.0825 \\
& $n=7$ & 1.0549 & 0.9480 & 0.5537 & 0.4463 & 3.8194 & 43.806 & 0.0831 \\
Long correlated & $n=8$ & 1.0513 & 0.9512 & 0.5502 & 0.4498 & 3.9639 & 43.980 & 0.0834 \\
binomial model & $b_{i}=9 / 10^{9}$ & & & & & & & \\
& and & 1.0513 & 0.9512 & 0.5502 & 0.4498 & 4.6204 & 44.153 & 0.1335 \\
& $n=8$ & & & & & & & \\
\hline
\end{tabular}

(Note: 3th to 9th form is an important parameter required for the various models, if there is not use various we use "-" indicates)

The following are the proposed long-range dependence in the binomial model when $n=6, n=7, n=8$ and $n=8, b_{i}=9 / 10^{9-i}$ the corresponding random variable (abscissa value) and probability (ordinate values).

Table 2.1 shows that the classic binary model tends to the $B-S$ formula, and the conclusion is consistent and convergent. And the literature [8-9] also gives the proof. But the variance of former will increase and will be larger than the latter. Because the correlation structure of the long binomial model is built on the top of a binary tree, and the special nature of construction. They make random variable factorial growth was observed data can be found in close proximity to the conclusion of the $B-S$ formula. And we can be seen from the above chart, when $n$ is increasing its distribution of random variables is tend distribution. Since we assume $b_{i}=2^{-(n-i+1)}$, the convergence has weakened. The last behavioral assumptions is $n=8, b_{i}=9 / 10^{9-i}$ in table 2 , we get figure 3.4distribution. From the chart we can find out that the choice of $b_{i}$ will direct impact on the pricing of stock options and are consistent, and it also is convergent with the formula. Contact for specific performance of the three models, when, $b_{n}=1, b_{i}=0 \quad(i \neq n)$ this model is the binomial model, when $n \rightarrow \infty$, the model and the $B-S$ model have same result. So we can conclude that classical binomial model and the $B-S$ model is one of the special circumstances related to the proposed length of the binary model.

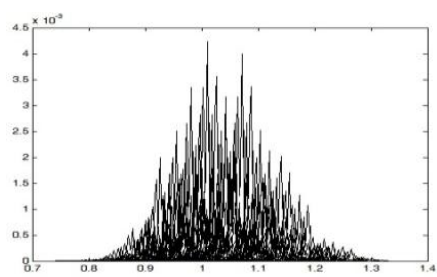

Fig. 3.1: $n=6$

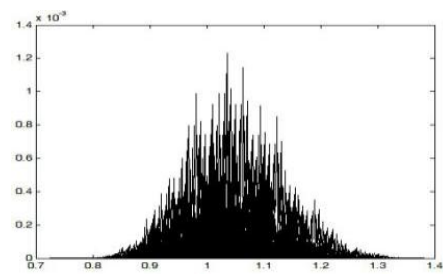

Fig. 3.2: $n=7$ 


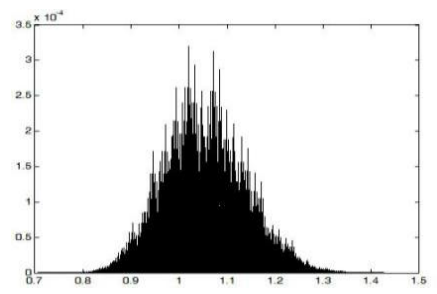

Fig. 3.3: $n=8$

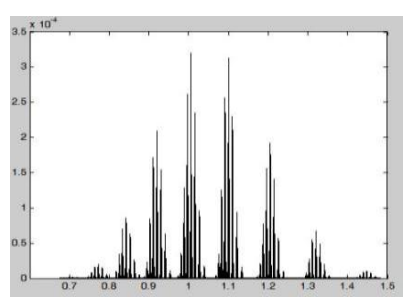

Fig. 3.4: $n=8, b_{i}=9 / 10^{9-i}$

\section{Summary}

Suppose all investors look the same to historical stock prices on the future price of the right degree of influence weight, and investors understand the long-related contact with the historical price of the stock price are different. This paper established a long, complex process of the stock price. We have compared three model, we know that Classical binomial model and $\boldsymbol{B}-\boldsymbol{S}$ formula are special cases of the long correlation binary model. So this stock option pricing model has universal significance. So it provides an important reference to other derivatives pricing.

\section{Reference}

[1] Cox, J. C., S. A. Ross, and M. Rubinstein. Option Pricing: A Simplified Approach [J]. Journal of Financial Economics 7(October 1979):229-64.

[2] Fischer Black and Myron Scholes, The Pricing of Options and Corporate Liabilities [J]. Journal of Political Economy,81 May-June,1973:637-654.

[3] John C. Hull. Options, futures and other derivatives[ M].Mechanical industry press,2011.

[4] Shreve, S.E. Stochastic calculus for finance [M]. Shanghai university of finance and economics press,2008.10.

[5] FU De-wei. Random binominal option pricing model and Simulation[D]. Xiamen university,2008.

[6] YANG Shao-hua. The pricing model of European option binary tree improvement and empirical research[J]. Journal of Jilin institute of chemical industry, 2012,07:92-94.

[7] WANG Jun-wei, Luo chun. Binary-tree Model Based on Bayesian[J]. Journal of Shanghai institute of applied technology (natural science edition), 2009,02:119-121.

[8] YAN Xin. The convergence of the binary tree option pricing model[D],Jilin university,2008.

[9] QU Jun-heng, ZhANG Zhan-ying, Zhao Chun-ru. The limited situation of Binomial trees model in the Option Pricing [J]. Journal of Xuchang university,2007, 05: 1-5. 